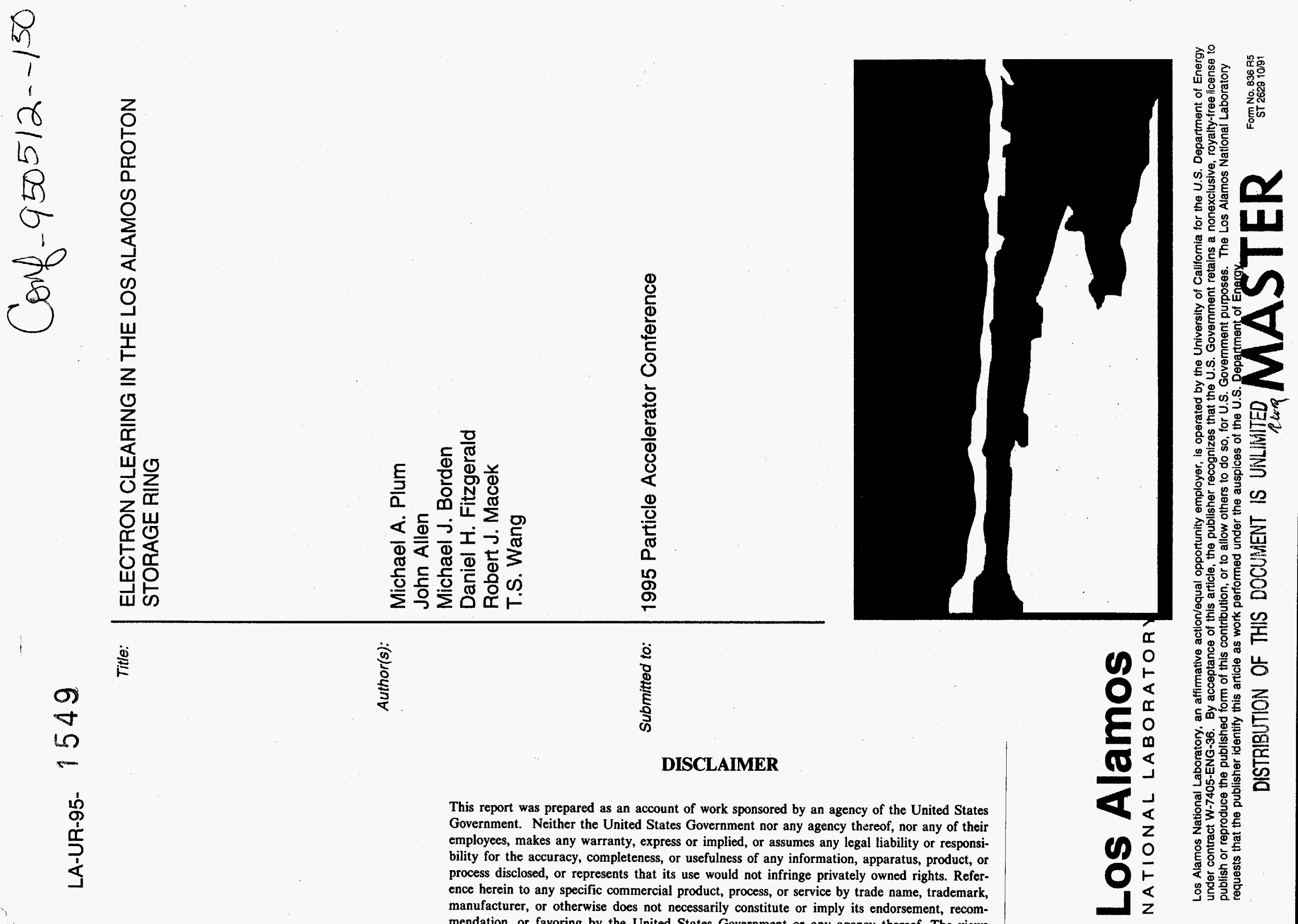

mendation, or faving by the United States con mendation, or favoring by the United States Government or any agency thereof. The views
and opinions of authors expressed herein do not necessarily state or reflect those of the United States Government or any agency thereof. 


\section{DISCLAIMER}

Portions of this document may be illegible in electronic image products. Images are produced from the best available original document. 


\title{
ELECTRON CLEARING IN THE LOS ALAMOS PROTON STORAGE RING
}

\author{
M. PLUM, J. ALLEN, M. BORDEN, D. FITZGERALD, R. MACEK, T.S. WANG \\ Accelerator Operations and Technology Division \\ Los Alamos National Laboratory, Los Alamos, NM 87545
}

\begin{abstract}
The instability observed in the Los Alamos Proton Storage Ring (PSR) has been tentatively identified as an electronproton instability. A source of electrons must exist for this instability to occur. The PSR injection section contains the stripper foil, and therefore provides several strong sources of electrons. We have installed an electron clearing system in the injection section to clear out these electrons. The system comprises: 1) a foil biasing system to clear the SEM and thermionic electrons, 2) a pair of low-field bending magnets with a Faraday cup to clear the convoy electrons, and 3) two pairs of clearing electrodes, one upstream and one downstream of the stripper foil, to clear the remaining electrons. In this paper we will discuss the design and performance of the Electron Clearing System, and its effect on the instability. We will also present some results from other charge-collection experiments that suggest there is also substantial electron production in parts of the ring other than the injection section.
\end{abstract}

\section{INTRODUCTION}

Electrons are an important issue for the PSR because we suspect our instability is an e-p instability. In this instability, background low-energy electrons are trapped in the spacecharge potential of the proton beam. Above a certain threshold, the electrons and protons develop coupled oscillations that grow in time, eventually leading to beam loss. The biggest sources of electrons are probably in the injection section, which contains the $16-\mathrm{mm} \times 16-\mathrm{mm} \times 200 \mu \mathrm{g} / \mathrm{cm}^{2}$ carbon stripper foil. Because of this foil, we have [1] electrons due to secondary emission (SEM) from the stripper foil, "convoy" electrons stripped from the incoming $\mathrm{H}$ beam, thermionic electrons from energy deposition in the stripper foil, and knock-on electrons from interactions of the circulating and injected protons with the stripper foil. We also have residual-gas ionization and SEM from any beam-pipe surfaces the proton beam may interact with. The SEM and thermionic electrons have low energies (a few eV), the convoy electrons have kinetic energies of $430-\mathrm{keV}$, and the knock-on electrons have kinetic energies that range from a few $\mathrm{eV}$ to $2.4 \mathrm{MeV}$. For each $\mathrm{H}^{0}$ particle injected into the ring, we expect from the foil 1.0 convoy electrons, 3.6 SEM electrons, 1.2 knock-on electrons, and less than 0.002 thermionic electrons. We also expect about 0.0074 electron-ion pairs created over the length of the section from residual-gas ionization.

\section{ELECTRON CLEARING SYSTEM}

To control these electrons we built three subsystems, illustrated in Fig. 1: 1) clearing electrodes (two pair) for the ions and electrons from residual gas ionization, and the electrons from SEM off the beam-pipe walls caused by beam halo scraping; 2) a stripper-foil-biasing system to control the thermionic and SEM electrons from leaving the foil; and 3) a bending magnet and Faraday cup for the $430-\mathrm{keV}$ convoy electrons. We will now briefly discuss each of these three subsystems.

\section{CLEARING ELECTRODES}

The purpose of the clearing electrodes is to clear the charged particles caused by residual-gas ionization and the SEM electrons caused by the beam halo scraping on the beam-pipe walls. These electrodes must create an electric field (E-field) strong enough to overcome the E-field due to the space charge of the beam. In high-intensity machines, these space-charge fields can be considerable. For the PSR the maximum spacecharge electric field is about $250,000 \mathrm{~V} / \mathrm{m}$. We installed two pair of clearing electrodes, one upstream and one downstream of the stripper foil. Our aluminum electrodes are curved to match the cross section of the beam pipe, and each one covers 90 degrees of the circumference. Each electrode can be biased up to $30 \mathrm{kV}$. The electrodes upstream of the stripper foil are separated by $10 \mathrm{~cm}$, and the electrodes downstream of the stripper foil are separated by $14 \mathrm{~cm}$. These separations correspond to maximum E-fields of $600,000 \mathrm{~V} / \mathrm{m}$ and $430,000 \mathrm{~V} / \mathrm{m}$, respectively.

\section{STRIPPER-FOIL BIASING}

The purpose of the stripper-foil biasing subsystem is to control the SEM, thermionic, and low-energy knock-on electrons emitted from the foil. We cannot simply use clearing electrodes or clearing rings for this purpose, since the foil is suspended in the center of a metal frame, and the E-field created by the electrodes would be strong between the foil frame and the electrodes but weak at the center of the foil, where we need it the most. So we chose instead to bias the stripper foil. With our high beam intensities, the electric field at the center of the surface of the foil is about $1 \mathrm{MV} / \mathrm{m}[1]$, assuming a perfectly-conducting, full-aperture foil. We cannot bias it enough to prevent electrons from being pulled off the foil, but if we bias it below the depth of the beam's potential well $(\approx 10 \mathrm{kV})$, we can create a potential well 1 to $2 \mathrm{~cm}$ away 


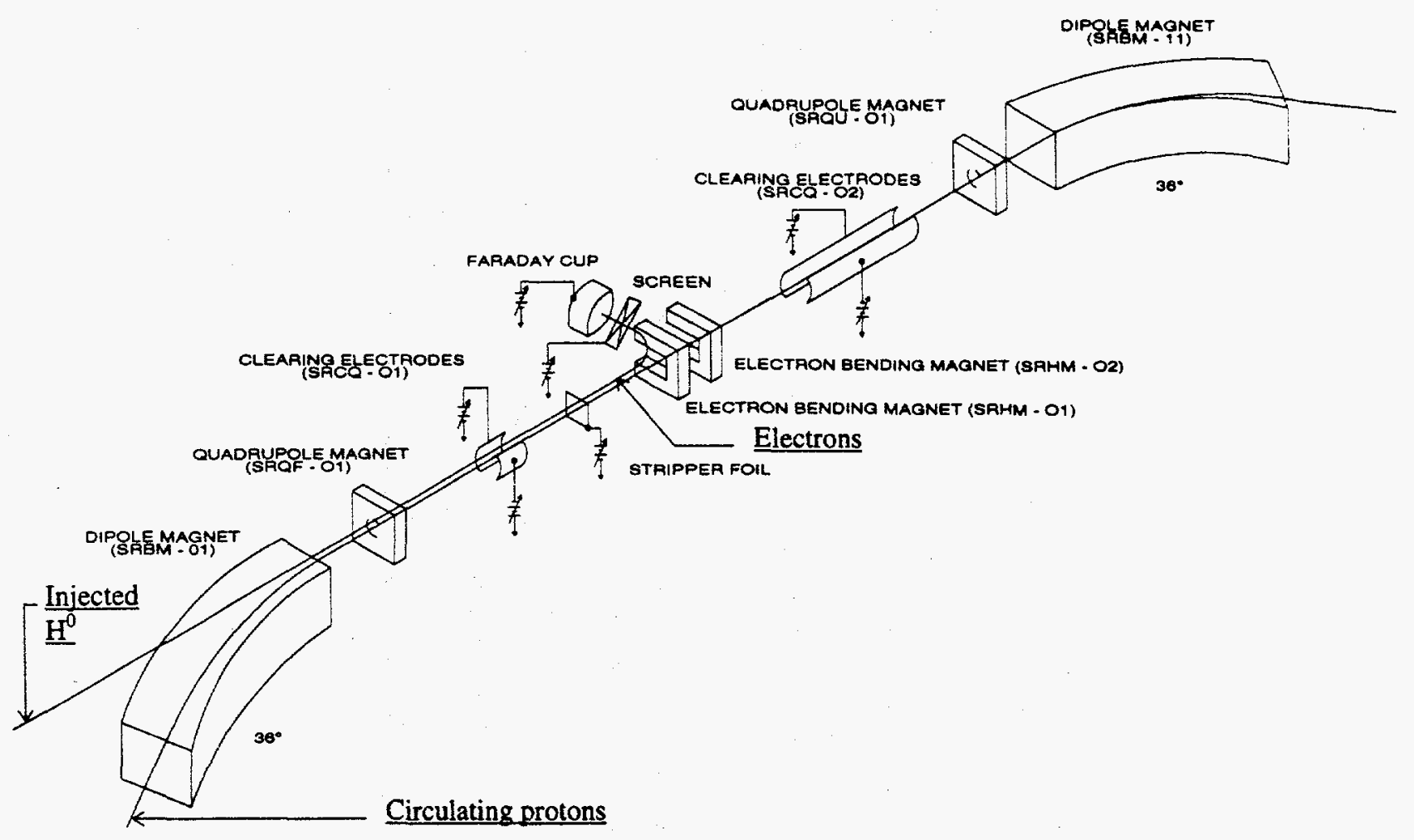

Fig. 1. A diagram of the Electron Clearing System.

from the foil to trap the electrons. Then, when the beam intensity drops at the end of the beam bunch the bias $E$-field will be strong enough to attract the electrons and reabsorb them. The electrons are therefore cleared after each revolution of the beam around the ring, or about every $360 \mathrm{~ns}$. We can bias the foil up to about $11 \mathrm{kV}$, limited by the insulating properties of the foil-mounting system.

\section{FARADAY CUP}

The purpose of the faraday-cup subsystem is to collect the $430-\mathrm{keV}$ convoy electrons. The energy of these electrons is too high to clear with electrodes, so we bend them $90^{\circ}$ with a low-field bending magnet into a Faraday Cup. A second magnet identical to the first, but opposite in polarity, and located just downstream of the first, cancels the closed-orbit distortion and the effects of the non-linear magnetic fields. We also installed a screen at the entrance to the Faraday cup, flush with the inside of the beam pipe, to avoid any wake-field effects. We can bias this screen to reduce the SEM effects when the convoy electrons hit the Faraday cup, and also to attract any low-energy electrons and negative ions from within the beam pipe. We found that a Faraday cup bias of $+200 \mathrm{~V}$, a screen bias of $-100 \mathrm{~V}$, and a magnetic field of 420 Gauss, is sufficient to clear the convoy electrons.

\section{WHAT ABOUT THE DELTA RAYS?}

The three subsystems discussed above deal effectively with all the electron sources but one - the delta-ray (or high-energy knock-on) electrons. These electron energies span the range from a few eV to $2.4 \mathrm{MeV}$. Most of the delta rays from the foil have low kinetic energy, and they will be trapped between the foil and the Faraday-cup magnets, and should eventually be cleared by the biased foil. The medium-energy delta rays will be deflected into the Faraday cup, and the few (about $3 \times 10^{-5}$ per proton) high-energy delta rays that pass through the Faraday-cup magnets will be lost on the beam-pipe walls. After commissioning the electron-clearing system in 1993, we believe we can collect almost all the electrons created in the PSR injection section (Section 0).

\section{THE RESULTS}

During machine development tests in 1993, we performed experiments with both bunched and coasting (dc) beams. Some examples of signals from the stripper foil are shown in Fig. 2. We found that the Electron Clearing System does a good job at effectively clearing the electrons. We found that we can raise the threshold of the coasting-beam instability by about $20 \%$, and increase the growth time of the bunched-beam instability by almost an order of magnitude (but not alter its threshold). The instability was not cured. We suspect that 


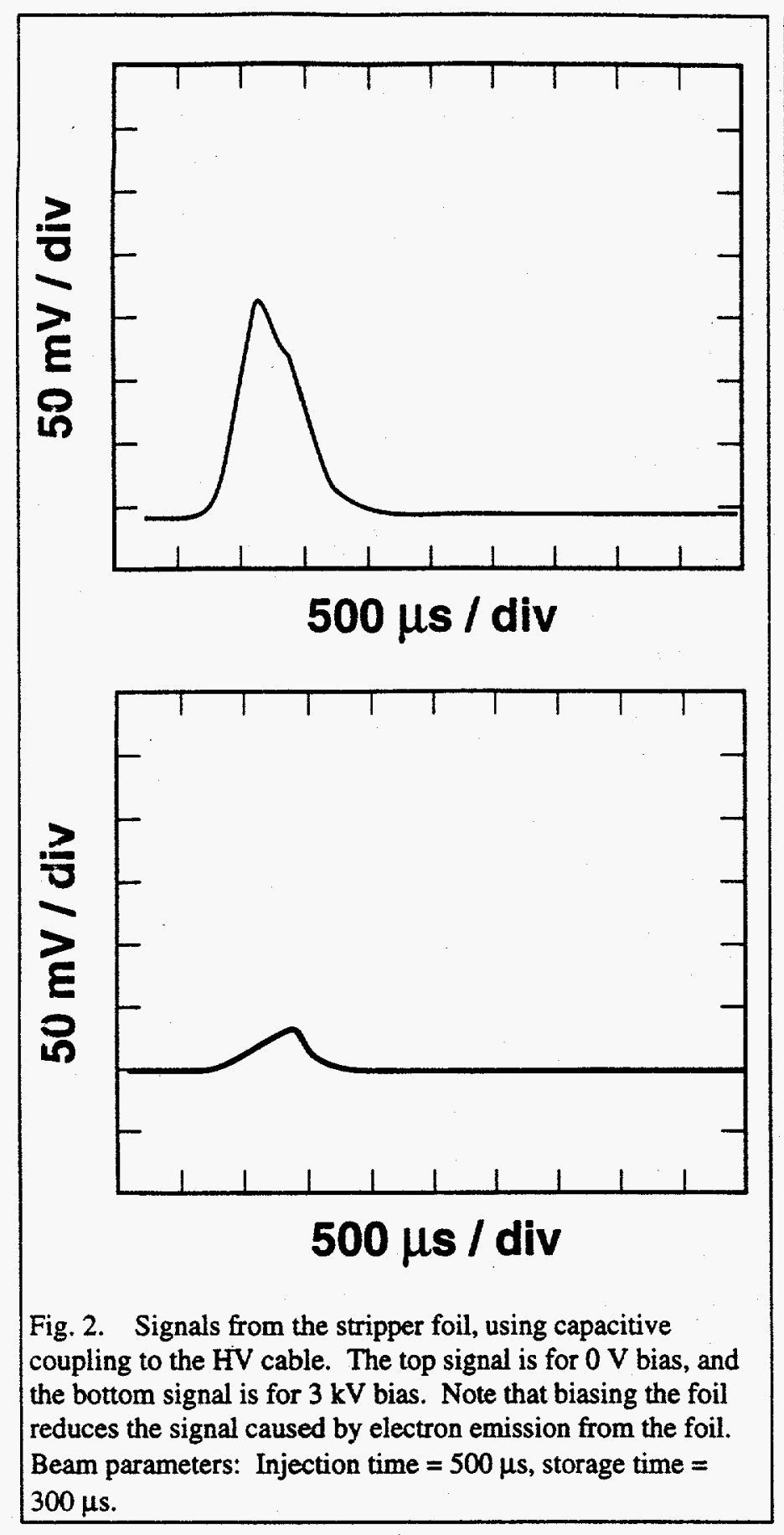

although we were able to clear the electrons from Section 0 , there are probably other strong sources of electrons in one or more other sections. To search for other sources, we have initiated a new set of experiments of identify and characterize them.

\section{OTHER SOURCES OF ELECTRONS}

In another section of the ring (Section 3) we have a pinger [2] with two 4-m long electrodes. By biasing the electrodes we can use the pinger as an ion chamber to collect charge from

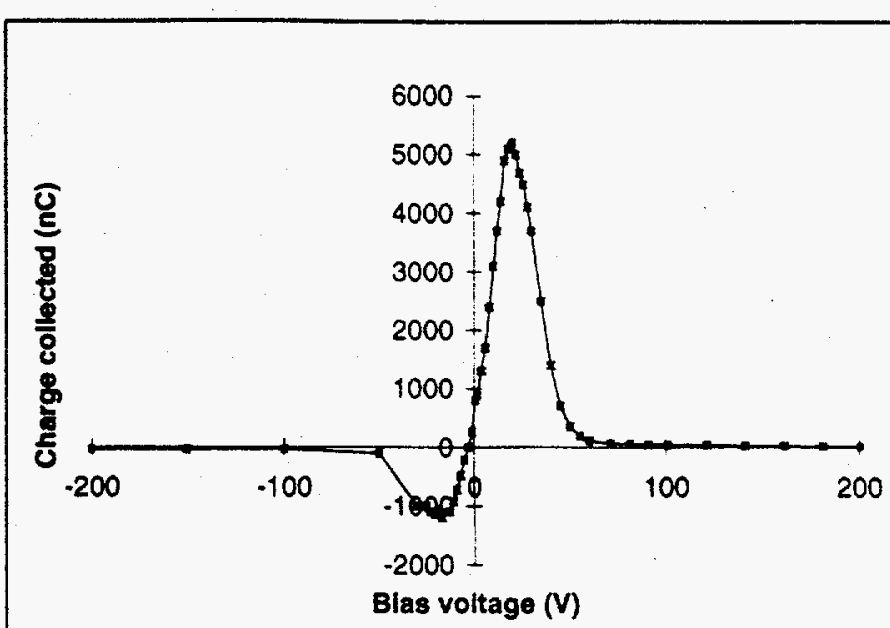

Fig. 3. An example of the charge collected from Section 3 of the PSR. This data was taken during production conditions, with a maximum charge of about $3.75 \mu \mathrm{C}$ in the ring. The data remain flat above $200 \mathrm{~V}$ and below $-200 \mathrm{~V}$.

within the beam pipe. Poisoning the vacuum at low beam intensities gives the result expected for residual gas ionization, but at higher beam intensities we have observed unusual and unexplained results for bunched beams. An example [3] is shown in Fig. 3, where a small change in the electrode biasing makes a big change in the amount of charge collected. In another case, with the same setup, we collected charge equivalent to $25 \%$ of the total beam charge in the ring!

\section{SUMMARY}

The Los Alamos Proton Storage Ring has an instability at high beam intensities that we have tentatively identified as an e-p instability. We built an Electron Clearing System to attempt to cure the instability. We found that although we effectively cleared the electrons from the most prolific section of the ring, we made only small improvements in the instability threshold. We have identified other, surprisingly strong, sources of electrons, and we are working to understand them.

[1] M. Plum, "Electric Fields, Electron Production, and Electron Motion at the Stripper Foil in the Los Alamos Proton Storage Ring", 1995 IEEE Particle Accelerator Conference, these proceedings.

[2] T.W. Hardek and H.A. Thiessen, "A Pinger System for the Los Alamos Proton Storage Ring", 1991 IEEE Particle Accelerator Conference, IEEE Conference Record 91CH30387, p. 866.

[3] M. Plum et. al., "Pinger Ion Chamber Development", PSR Tech Note PSR-94-03, Los Alamos National Laboratory, June 22, 1994. 